\title{
Fast image stitching method for handling dynamic object problems in Panoramic I mages
}

\author{
Murodjon Abdukholikov ${ }^{1}$, Taegkeun Whangbo ${ }^{2}$ \\ ${ }^{1}$ Department of IT Convergence Engineering, Gachon University \\ Sujeong-Gu, Seongnam-si, Gyeonggi-Do, 461-701, Korea \\ [e-mail: m.abdukholiqov@gmail.com] \\ ${ }^{2}$ Department of IT Convergence, College of IT, Gachon University \\ Sujeong-Gu, Seongnam-si, Gyeonggi-Do, 461-701, Korea \\ [e-mail: tkwhangbo@gachon.ac.kr] \\ *Corresponding author: Taegkeun Whangbo
}

Received March 14, 2017; revised May 9, 2017; accepted July 20, 2017; published November 30, 2017

\begin{abstract}
The construction of panoramic images on smartphones and low-powered devices is a challenging task. In this paper, we propose a new approach for smoothly stitching images on mobile phones in the presence of moving objects in the scene. Our main contributions include handling moving object problems, reducing processing time, and generating rectangular panoramic images. First, unique and robust feature points are extracted using fast ORB method and a feature matching technique is applied to match the extracted feature points. After obtaining good matched feature points, we employ the non-deterministic RANSAC algorithm to discard wrong matches, and the hommography transformation matrix parameters are estimated with the algorithm. Afterward, we determine precise overlap regions of neighboring images and calculate their absolute differences. Then, thresholding operation and noise removal filtering are applied to create a mask of possible moving object regions. Sequentially, an optimal seam is estimated using dynamic programming algorithm, and a combination of linear blending with the mask information is applied to avoid seam transition and ghosting artifacts. Finally, image-cropping operation is utilized to obtain a rectangular boundary image from the stitched image.

Experiments demonstrate that our method is able to produce panoramic images quickly despite the existence of moving objects.
\end{abstract}

Keywords: Image stitching, moving object, moving object detection, difference of overlap regions, image cropping, ghosting artifacts

A preliminary version of this paper appeared in the $1^{\text {st }}$ International Conference on Emerging Trends \& Innovation in ICT(IEEE conference, Pune Section), February 3-5, 2017, Pune, India. This work was supported by Institute for Information \& communications Technology Promotion(IITP) grant funded by the Korea government(MSIP)(No.2017-0-00180, Development of Complex biosignal response information based intelligent VR(Virtual Reality) life care technology) 


\section{Introduction}

Image stitching algorithms have been researched for many years. They are useful in different fields of computer vision, computer graphics, virtual reality and others. Presently, There are many stitching algorithms and hardware devices for creating panoramic images. However, a variety of problems exists, such as ghosting could probably seen in panoramic images[1]. We take a sequence of images to create a panoramic image. The availability of moving objects, which appear in different positions of neighboring images will cause the ghosting artifacts in the overlapping areas between images [2].

Additionally, the use of image stitching algorithms in low-powered devices and real-time applications is a challenging task [3] because most of the image stitching methods are computationally expensive. Finding the corresponding feature points from images and matching them with feature extractor methods as well as feature matching techniques, such as SIFT[4], SURF[5] and ORB feature extraction methods [6] take so much time and consume huge free memory space on devices. Therefore, the stitching algorithm on the computer must be tailored to satisfy the real-time requirements of mobile phones with limited computational resources [7].

Furthermore, due to capturing image sequences with different rotational angles, transforming images into the same coordinate space with various projection techniques most of image stitching applications construct panoramic images with irregular boundaries. However, users prefer to have a rectangular boundary of panoramic images while they share these panoramic images on social networks as well as store them on smartphones. Therefore, we have to take into account the production of panoramic images with rectangular boundaries.

The main contribution of this work is that we present a fast and automatic image stitching method for mobile phones, which creates pleasing and physically consistent panoramic images with low computational time despite the presence of moving objects in the scene. Our proposed system consists of the following stages:

- Generating feature points based on ORB;

- Finding correct feature matches using FLANN algorithm and removing outliers using RANSAC (Random Sample Consensus) method;

- Determining a precise overlap region between neighboring images;

- Calculating the difference between the overlap regions by applying thresholding and median filtering;

- Finding an optimal seam in the overlap region of images;

- Applying image blending and image cropping techniques;

The remaining part of the paper is divided into several sections: Section 2 presents an overview of some related image stitching studies. Section 3 provides detailed discussion on our proposed image stitching method for handling moving object problems. Section 4 demonstrates our experimental results and analysis. Finally, we draw conclusions based on the experimental results in Section 5.

\section{Related Work}

Image Stitching has been investigated over many years and many scientists and researchers are commonly working on image stitching domain to reduce processing time and improve the quality of image mosaics. Yanli Wan et al [8] proposed an image stitching method for moving 
objects to eliminate ghosting artifacts. In their method, the blending area is rearranged to avoid blending the moving object in the scene. However, this method is not easy to use in the general case. JunWei Hsieh [9] proposed an edge-based method for stitching a series of images. In this approach, for purpose of robustness consideration, the initial estimate used two different schemes: the edge-alignment and the correspondence-based. Finally, a Monte- Carlo style method is proposed to find the best motion parameters by integrating these two methods.

Jisung Yoo et al [10] proposed a regional linear warping based image stitching method. Initially, the method utilizes dominant edge information for approximating input images as multiple planar surfaces, and each planar surface is warped relatively with different hommographies. This method can stitch images with a large parallax successfully but the process of estimating multiple hommographies, searching seed points and propogating them for dominant edges consumes so much time. Similar work to Jisung Yoo's work is adaptive as-natural-as-possible image stitching method which was proposed by Chung-Ching Lin et al[11]. The method employs the global similarity transformation with the combination of local hommography. To reduce perspective distortion in the non-overlapping areas, a local hommography is linearized and it is changed slowly to global similiraty transformation. This approach also can handle parallax, perspective distortion artifacts, however image fusion algorithms should be integrated for seamless blending and processing time should be reduced. Qingpeng Chai et al [12] proposed a novel weight-based shape-optimizing warping framework to reduce projective distortion artifacts. They aligned input images with the projective transformation and the similarity transformation, as a result, the projective distortion is reduced on the panoramic image. As the demand for panoramic images grows, image stitching applications on mobile phones and low-powered devices become popular. Xiaomin Ma et al [13] presented a fast affine-invariant features based- stitching method for low-powered devices and mobile phones. AFREAK [14] method is employed to extract feature points and Hamming distance method is applied for feature matching instead of Euclidean distance. The speed of producing panoramic image is reduced compared to other SIFT, SURF based- methods. But this method can not achieve good result when moving objects exist in the scene and this method causes the ghosting artifacts.

A new method for dynamic objects and blending based on motion prediction is proposed by Yu-jing Zhao et al [15] which handled ghosting effects. This algorithm shows good performance and provides a robust and pleasing result for video stitching. However, their method is inapplicable on mobile phones, because they used a fixed moving camera with constant speed to stitch images and deliver the result to surveillance applications. According to James Davis [16], the image mosaic is segmented into disjoint regions and the best ghosting regions are found using his method. However, this method is difficult to employ when many image pairs are overlapping in the same region. The closest method to Davis's work is an optimal seam selection based method proposed by Mills and Dudek [17]. They proposed to choose an optimal seam by applying the algorithm of Dijkstra [18] in the presence of moving objects and a mask is created by an optimal seam. These two disjoint regions are blended by this obtained mask along an optimal seam line, which produces a practically good result, but it is inapplicable for low-powered devices in real-time cases.

Yingen Xiong et al [2] proposed a gradient domain approach to address ghosting problems in the composite image. The Poisson equation is calculated with the boundary condition to recover the modified composite image. Using this method, ghosting artifacts are removed successfully, but large ghosting shapes are time-consuming to remove.

Gradient image blending approaches [19], [20], [21], [22], [23] have been widely used in image blending and editing. A new gradient vector field is created with the gradients of the 
source images. The new composite image can be recovered from the gradient vector field by solving the Poisson equation. An attractive result can be obtained by using this method, but it is a time-consuming process for large images. Multi-band blending algorithm which uses Laplacian pyramid scheme, is the most commonly used method [24], [25]. Images at each level of the pyramid are merged to produce a final result. Futhermore, one of the important steps of image stitching process is seam estimation. With the optimal seam, the overlap region of the image pairs is split into two regions and the overlap region is updated by merging them along the seam. An optimal seam helps to avoid cutting through moving objects in the overlap regions and produces a satisfactory result [26]. Many researchs are aimed at solving the seam estimation problem, such as Dijkstra algorithm, seam estimation by dynamic programming [27], [28], and graph cuts[29]. Among the seam estimation algorithms, the dynamic programming approach is faster.

We discuss another component of image stitching methods. One of the widely used feature extraction methods is SIFT, which has been proven remarkably successful in numerous applications. However, this method spends a lot of time to extract feature points. Therefore, it is not suited for real-time applications and low powered devices despite the aim of other studies to speed up the computation of SIFT, most notably with GPU-based devices [30]. On the other hand, Ethan Rublee et al [6] proposed the ORB method which is an efficient and robust feature extraction method suited for low-powered devices, mobile phones and real time applications. It uses an improved FAST (Features from accelerated segment test) [31] algorithm for finding feature points and BRIEF [32] binary descriptor. It is free for research and commercial usages and faster than SIFT and SURF.

\section{Proposed Method}

In this section, we discuss our proposed method in detail. Fig. 1 shows all steps of the method for creating a panoramic image.

Our proposed image stitching method has been integrated into traditional panoramic image construction method and it is capable of producing a panoramic image in two modes, it can construct a panoramic image in real-time or stitch already stored image sequences. To make our system work in real time, an image frame that can be read from a camera per second is delivered to the system. In the following subsections, we discuss all steps of the method.

\subsection{Extracting feature points and Matching}

Fig. 1 shows that, the first step of image stitching is Feature Extraction. In this step, we find the robust and unique feature points from the source images and compare image pairs to determine the matching relationship between input images. The extraction of feature points step includes detecting feature points and generating feature descriptors. In our proposed system, we employ the ORB feature extractor method because it consumes less memory space for generating feature points and it is faster compared to other feature detector methods.

In ORB [6], an improved FAST method with a circular radius of 9 is employed to detect feature points. First, for finding M number of feature points, a small threshold value is set to obtain more than $\mathrm{M}$ number of feature points. To find multi-scale corner feature points, the Harris corner detector is utilized at each level in the pyramid to discard key points along the edges. 


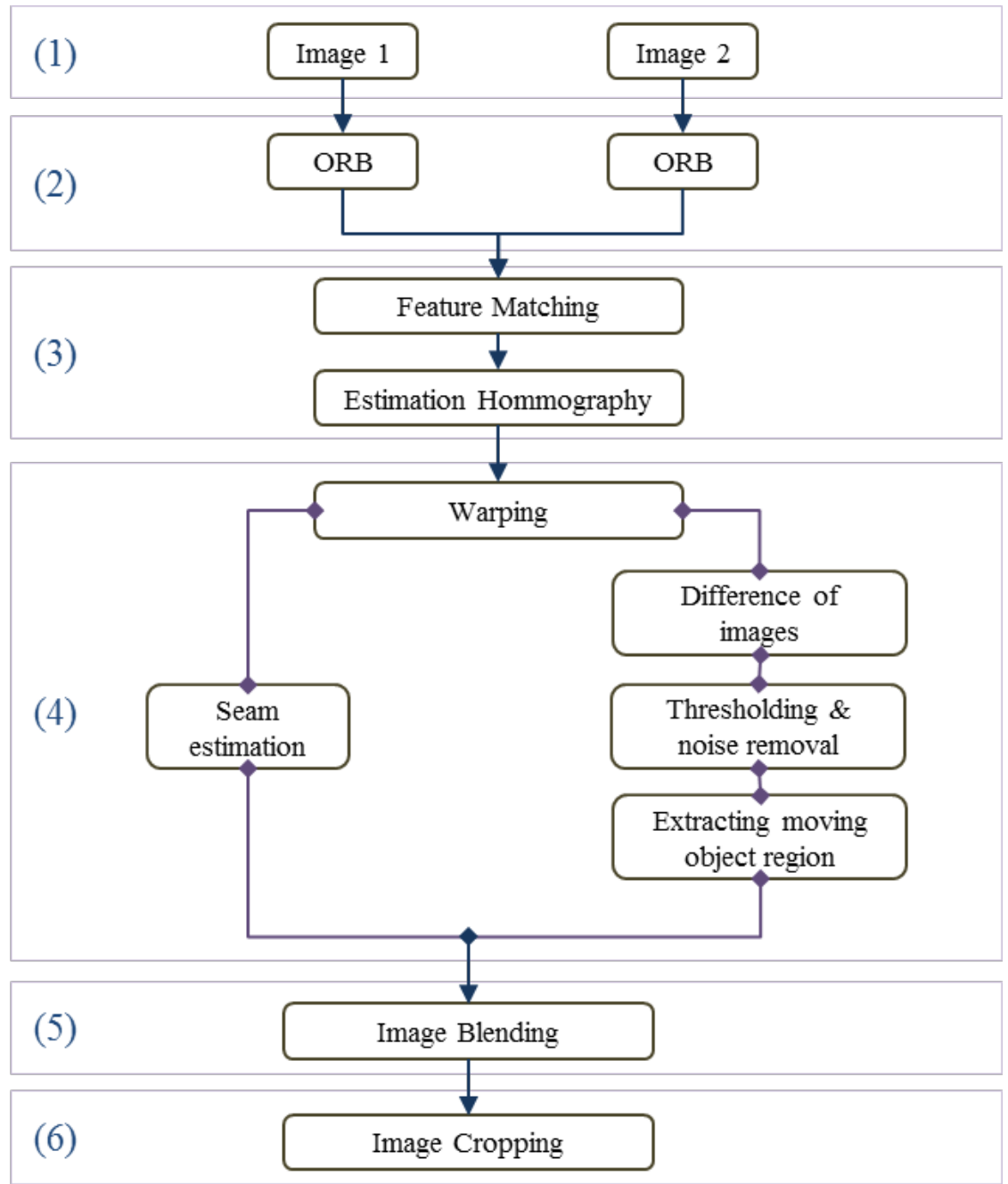

Fig. 1. Flowchart of the proposed method, 1) input images, 2) Feature extraction based on ORB, 3) Image registration, 4) Image correction, 5) Image blending, 6) Image cropping for rectangling images

The improved FAST method utilizes intensity centroid to determine the orientation of the corner [33], which can be found using standard moments and by defining the moments using:

$$
m_{p q}=\sum_{x, y} x^{p} y^{q} I(x, y)
$$

the centroid is calculated by:

$$
C=\left(\frac{m_{10}}{m_{00}}, \frac{m_{01}}{m_{00}}\right)
$$

and the patch orientation is found using:

$$
\theta=\operatorname{atan} 2\left(m_{01}, m_{10}\right)
$$


Once, all feature points have been detected with oriented FAST feature detection method, rBRIEF binary descriptor is used to generate binary feature descriptors from multi-scale oriented feature points. It is modified method of BRIEF binary descriptor. To increase the rotation invariance of the method, it is steered in accordance with the orientations of key points:

$$
S_{\theta}=R_{\theta} S
$$

where $R_{\theta}$-is the rotation matrix; $S$ - is a set of binary tests matrix; $S_{\theta}-$ is a steered version of $S$;

Then, a learning method has been developed to recover from the loss of variance in steered BRIEF and decrease the correlation to each of the binary tests. An applicable subset of binary test has been selected by utilizing the learning method.

After obtaining the corresponding ORB feature descriptors, image pairs are prepared to match. In our proposed system FLANN based Matcher can be utilized to match two images using the Hierarchical K-means Tree for generic feature matching. Fig. 2 demonstrates the extraction of ORB feature points and feature matching process.

\subsection{Estimation Hommography}

The next step of the image stitching algorithm is the estimation of hommography matrix using RANSAC algorithm. RANSAC is a non-deterministic method for estimating certain mathematical model parameters because it does not guarantee to return adequate model parameters. The method is used to discard wrong matches and to increase the accuracy of registration by choosing the closest match between two images with the help of separation of inliers and outliers. First, it involves randomly selection of minimal sample sets from previously generated feature points and mathematical model parameters are calculated from the elements of randomly selected minimal sample sets. Following that, all elements of input set are checked by RANSAC. If elements are consistent with the parameters of the model of the first step, they are kept as inliers of the set by a certain threshold value, and hommography matrix is recomputed from the inliers set. Fig. 2 (d) shows the matches after discarding outliers.

By utilizing the RANSAC algorithm, we can tally the hommography matrix of the overlapping region in concordance with those correct matching point pairs:

$$
H=\left[\begin{array}{lll}
h_{1} & h_{2} & h_{3} \\
h_{4} & h_{5} & h_{6} \\
h_{7} & h_{8} & 1
\end{array}\right]
$$

If we have two correctly matched points $m=(x, y), n=\left(x^{\prime}, y^{\prime}\right)$. According to the projective matrix, the relationship of these two points is:

$$
\left[\begin{array}{l}
x^{\prime} \\
y^{\prime} \\
1
\end{array}\right] \sim\left[\begin{array}{lll}
h_{1} & h_{2} & h_{3} \\
h_{4} & h_{5} & h_{6} \\
h_{7} & h_{8} & 1
\end{array}\right]\left[\begin{array}{l}
x \\
y \\
1
\end{array}\right]
$$

We can write the relationship between $m$ and $n$ using:

$$
x^{\prime}=\frac{h_{1} x+h_{2} y+h_{3}}{h_{7} x+h_{8} y+1}, y^{\prime}=\frac{h_{4} x+h_{5} y+h_{6}}{h_{7} x+h_{8} y+1}
$$




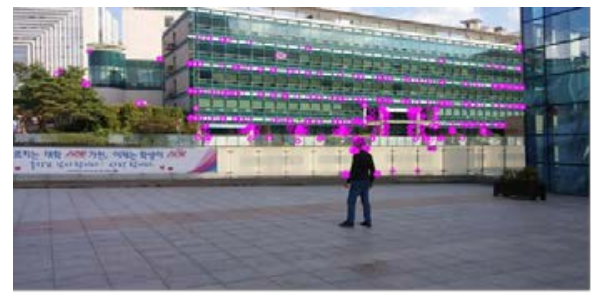

(a)

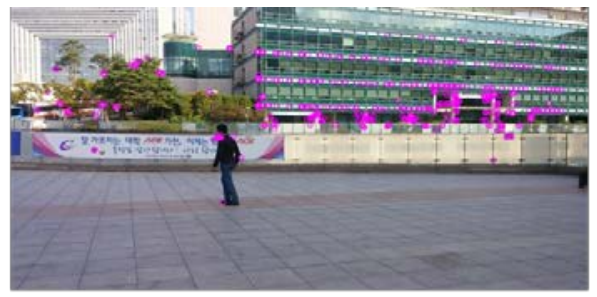

(b)

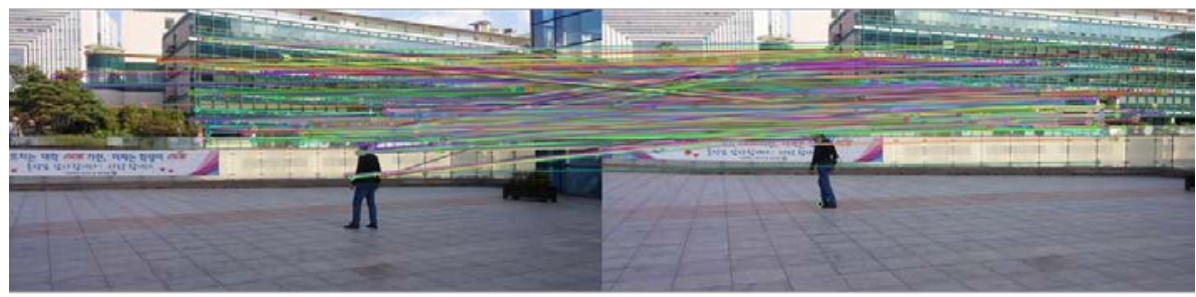

(c)

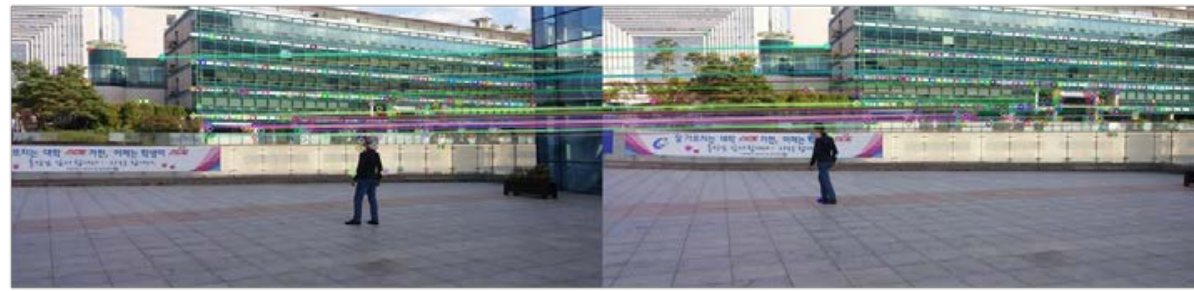

(d)

Fig. 2. Extraction of feature points (a) and (b), feature matching (c) and good matched feature points (d).

\subsection{Warping and blending}

After registering all input images with regard to each other successfully, we have to discuss how to create the final composite image because there are planar, cylindrical, spherical surfaces. When all registered input images are warped onto the final coordinate space, the next stage is to remove the seam artifacts. An overlap region of image pairs is updated with labeling information after finding an optimal seam. The images are merged along the seam in the overlap region to avoid splitting dynamic objects and reduce exposure differences. Afterward, if a seam is still visible, blending algorithms are used to smoothen the transition between images to address the seam effects. Traditional blending algorithms are based on direct average method [30] which is one of the easiest for implementation and quick approach. However it does not take into account the value of each pixel in the overlap region of image pairs, which equal to the sums of each pixel value from the reference image and target image multiplied by the weight. Generally, it is the simplest approach and can provide a satisfactory result. On the other hand, the method causes the ghosting artifacts to appear in the existence of moving objects in the overlap regions of input images. A possible solution could be the removal of the moving objects from one image and the filling of the removal regions with other image pixels to be stitched relative to the object's regions, which can result to an applicable and visually pleasing result.. The basic idea of our approach is to create a mask for the moving object in the overlap region and use it while blending. 


\subsection{Image correction}

The aim of this step is to create a binary mask to identify dynamic objects and to find an optimal seam in the overlap region of neighboring images. To complete this step, several image processing operations will be done. First, all registered input images are mapped onto the same coordinate space using hommography matrixes:

$$
X_{2}=H \times X_{1}
$$

Where $X_{2}$ is a warped image, $H$ - is the hommography matrix, Fig. 3- describes finding hommography matrixes for each image sequences and $X_{1}$ is an aspiring warping image.

The next step of image correction is to identify the lowest energy seam in the overlap region of neighboring images because we employ an optimal seam to cut the overlap region of image pairs and to merge them along the seam. The sum of energy of each pixel along the seam is the lowest with respect to any other seam in the image and it is least likely to be perceptible through our eyes. Although there are many well-known seam estimation algorithms, we address the problem via dynamic programming, which is one of the commonly used and fast seam estimation algorithms. Dynamic programming algorithm finds a low-cost path by scanning the error surface (the squared difference). We calculate current pixel energy with the addition of the energy of one pixel from three possible pixels above it and this is mathematically defined as follows:

$$
e=\left(I_{1}-I_{2}\right)^{2}
$$

Where $e$ - is the squared difference of overlap regions; $I_{1}, I_{2}$-are neighboring mapped images;

$$
S_{i}(j)=e(i, j)+\min \left\{\begin{array}{l}
S_{i-1}(j-1) \\
S_{i-1}(j) \\
S_{i-1}(j+1)
\end{array}\right.
$$

Where $S_{i}(j)$ - is the total energy of the path, $e$ - is the enery of the pixel in $(i, j), i$ - is the current row of the image, $j$-is the current column of the image and min- denotes the minimum of three top neighboring pixel energies.

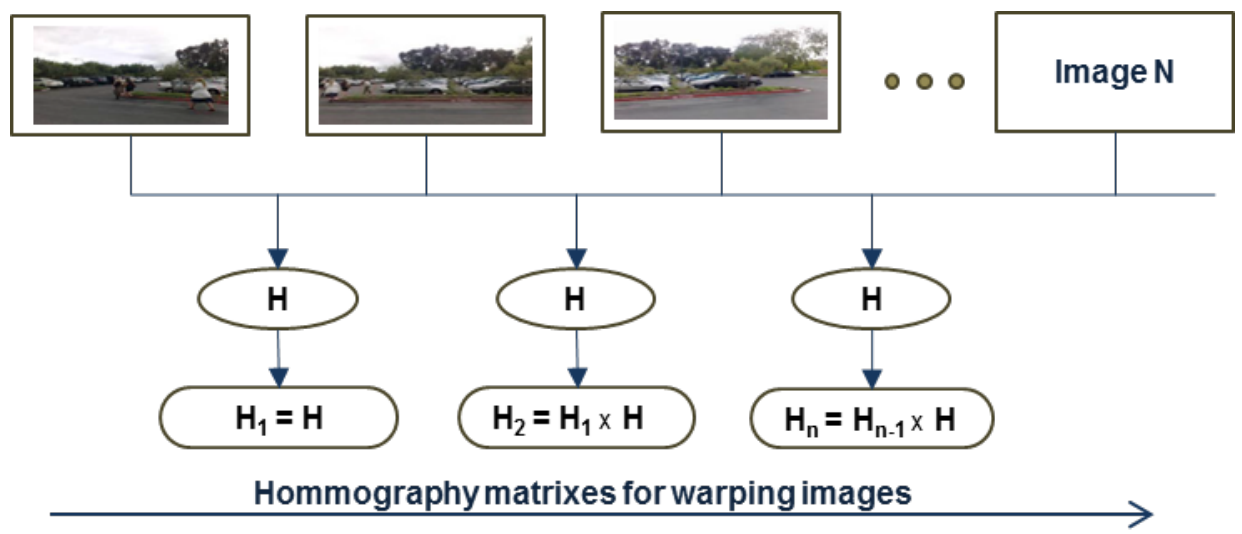

Fig. 3. Calculating the hommography matrix for each neighboring image pairs 
We compare the energy function of each pixel in the last row and choose the pixel that has minimum energy as the end of the optimal path. Following that, we obtain the optimal seam by tracing back the path of pixels with a minimum energy cost from bottom to top. After obtaining the optimal seam, we split the overlap region of images with the seam and update the overlap region by merging them along the seam. Fig. 4 demonstrates all steps of seam estimation.

To create a mask for moving objects in the overlap region, we calculate an absolute difference between the overlap regions of images.

$$
D(x, y)=\operatorname{abs}(I(x, y)-W(x, y))
$$

Where $D$ is the difference of images, $I(x, y)$ is a composite image at $(x, y)$ and $W(x, y)$ is a warping image at $(x, y)$;

As soon as the absolute difference of the overlap regions has been calculated, a thresholding operation is applied to the obtained image to segment the moving object region and the background region. This absolute difference information tells that how much these overlap regions are similar to each other. If a moving object appears in the overlap region of images, larger intensity differences can be seen while calculating the differences between them. Conversely, identical regions of the overlap have small intensity differences. We perform thresholding operation using:

$$
I(x, y)=\left\{\begin{array}{l}
1, \operatorname{abs}(I(x, y))>40 \\
0, \text { otherwise }
\end{array}\right.
$$

Image registration and large illumination changes may influence on segmenting moving object regions accurately. Prior to computing the absolute difference of overlap regions, the average of color differences is computed at the optimal seam and these values are distributed to the overlap region to decrease illumination changes. Unwanted edges and noises can appear on the difference of images in case the registration error is more than a pixel.

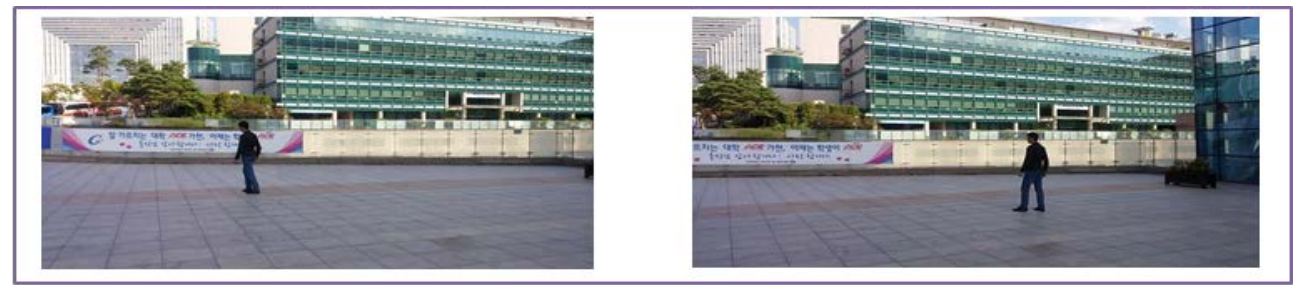

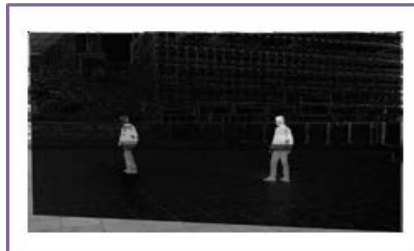

b) a)

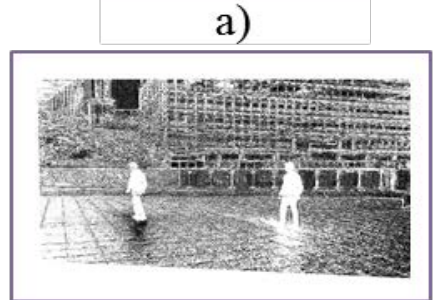

c)

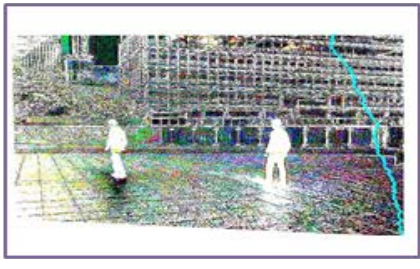

d)

Fig. 4. Seam estimation process. a) input images, b) absolute difference of the overlap regions, c) the squared difference of the overlap regions, d) an optimal seam for the region 
To address this problem, we apply the median noise removal technique to the derived binary image after completing the thresholding operation. As a result, we obtain the binary image(mask) of possible moving objects in the overlap region, and the mask information can be utilized with blending operations. Fig. 5- shows the obtained mask for moving objects in the overlap regions.

\subsection{Image blending}

Once all pixels of the overlap region have been updated along the seam, the next step is to apply the blending operation to avoid transition seams and producing a seamless attractive composite image. In our proposed system, we employ the combination of the simplest linear blending method with the mask information for dynamic objects. Feather, alpha blending methods combine two regions of the overlap linearly with weight values, and the sum of the weights for two pixels is one. Alpha blending degrades the boundaries of images in increasing and decreasing order using:

$$
I=I_{1} * \text { alpha }+I_{2} *(1-\text { alpha })
$$

Where $I_{1}, I_{2}$ - are two image pairs to be stitched; alpha- denotes the weight;

During the blending process, we use the left image pixels for the left side of the seam and vice verse to avoid updating the cut regions to the overlap region by the mask. Image regions are updated in the blending regions using mask information in addition to color difference at the seam while completing the linear blending. In order to compensate the color difference for the updated regions, we calculate the color differences at the seam and distribute it to the updated regions according to the position of images along the seam. In the next sections, we will demonstrate our results.

\subsection{Image cropping}

The final step of our proposed method is the process of rectangling a panoramic image. This step is also necessary for producing an attractive panoramic image with rectangular boundaries. Most of the stitched images have irregular boundaries caused by warping process with various projections, camera movements, and others. To avoid irregular boundaries, an automatic image-cropping technique is utilized to the final stitched image. From the information of hommography matrixes with warped images, the positions of the four corners of the stitched image are easy to find, and the minimum and maximum coordinate positions are

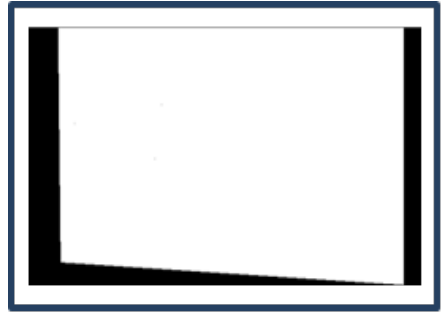

a)

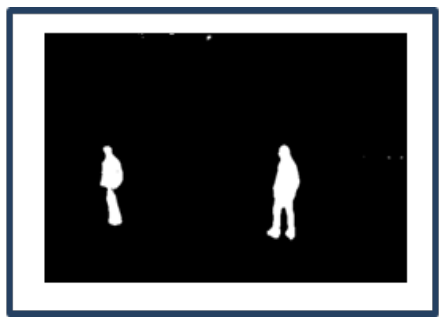

b)

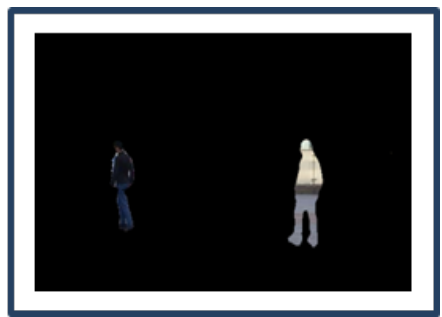

c)

Fig. 5. Creating a mask for moving objects. a) overlap region, b) a mask for moving objects, c) moving objects' regions using the mask. 


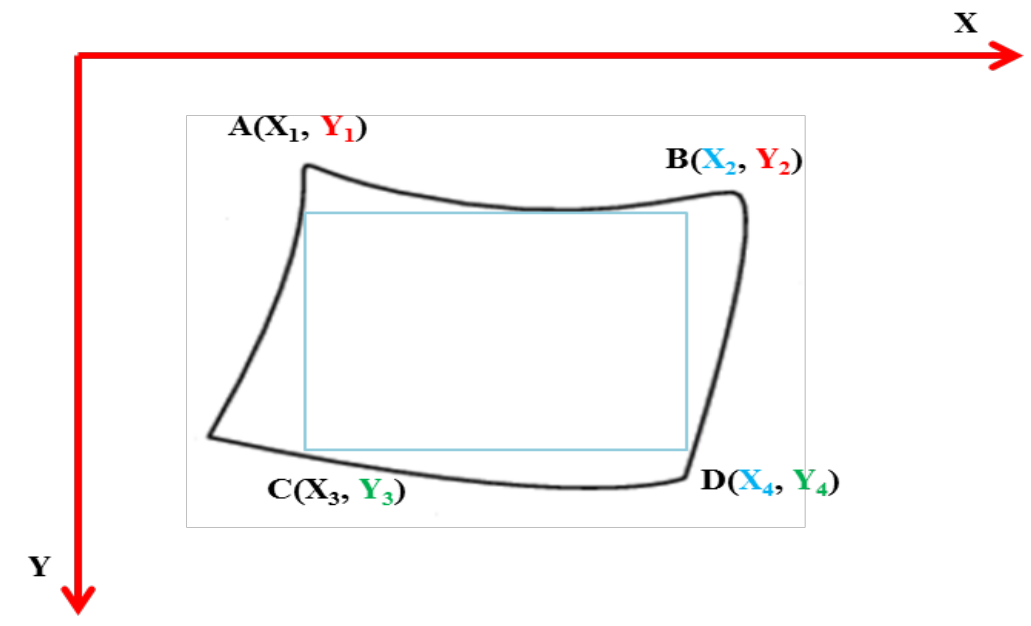

Fig. 6. Comparisons of the four positions of corners on the $\mathrm{X}$ and $\mathrm{Y}$ axis, which are denoted with the same colour. For example $\mathrm{Y}_{1}$ and $\mathrm{Y}_{2}$ values are common for A and B corners.

calculated with respect to both rows and columns. Full row and column based checking process is completed to determine four new corner coordinates of the panoramic image. Fig.6 demonstrates the automatic image-cropping process.

Furthermore, the manual image-cropping tool is provided unless a user is satisfied with the result of the automatic image cropping.

\section{Experimental Results and Analysis}

In this section, we demonstrate our experimental results to evaluate the performance of our method based on quality and speed. Furthermore, the comparisons between our method and the existing state-of-the-art methods are provided. To increase the speed of the image stitching process, we have utilized the methods that are fastest and suitable for low-powered devices, such as ORB feature detector, a seam estimation via dynamic programming, and a combination of linear blending with a mask information.

To check the quality of the stitched images, we compare our method with the popular commercial image stitching software and state-of-the-art methods, such as Auto Stitch (AS) [34], Chung-Ching's method (ANAP) [11], Xiaomin's method [13],and Panorama Maker (PM) [35].

Fig. 7 shows the stitched results produced by different methods. The result of our method appears to be attractive compared to the results of others. The moving object problems are adressed and seamless result is achieved using our method. In the final panoramic images of both AS and Xiaomin method ghosting artifacts appeared due to existence of a moving object. But the moving object problems are handled by applying PM, ANAP and the proposed method. ANAP produced stitching result without ghosting artifacts. It transformed one image on top of the second using global similarity and local hommography but transition seams can be seen clearly on the result of this approach. Furthermore, even though ghosting artifacts are eliminated by using PM, a walking person is doubled on the composite image and it looks unnatural. 
In many cases, both our method and PM perform well on handling dynamic object problems. Fig. 8 illustrates examples of stitched results in the presence of more than one moving objects in the overlap region of images. AS and Xiaomin methods failed to create panoramic images without ghosting artifacts. Because both of the methods do not have optimal searching technique and Xiaomin's blending method fails in the presence of moving objects.

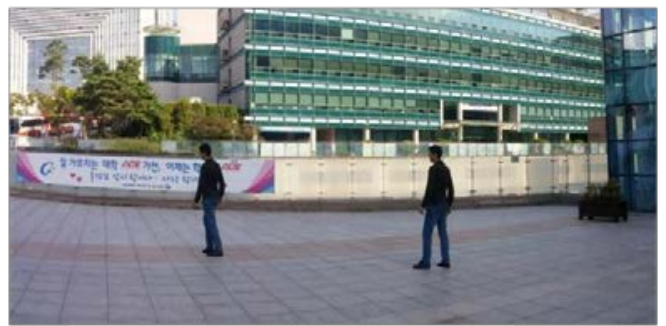

PM

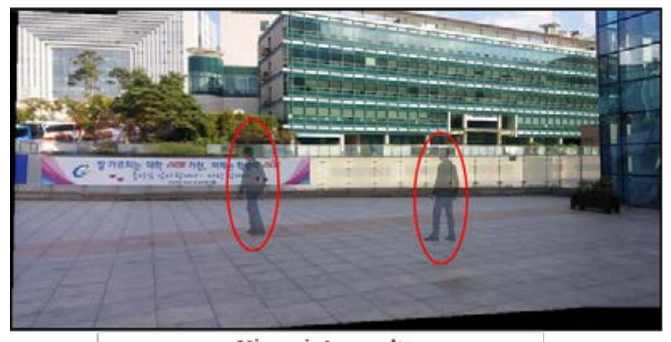

Xiaomin's result

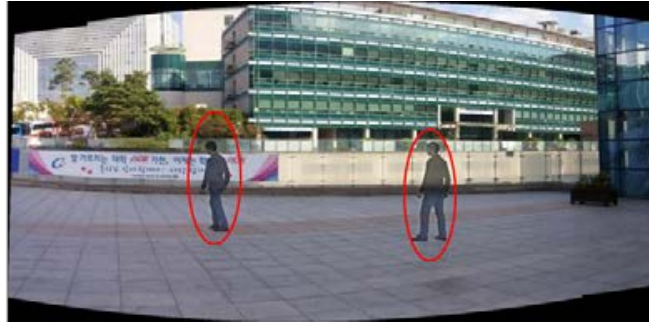

AS

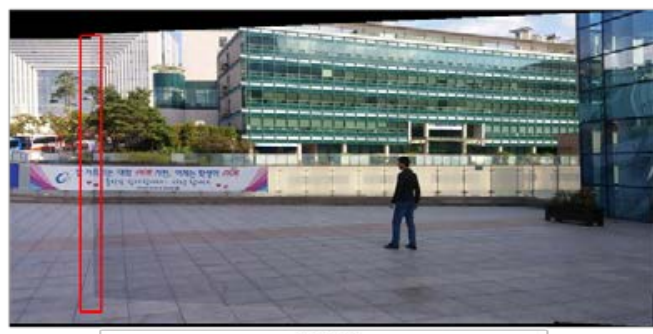

ANAP

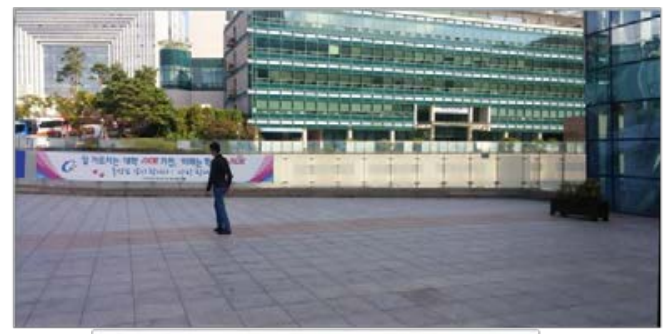

Our result

Fig. 7. Comparisons between state-of-the-art and our method's result, Panorama Maker (PM), Auto Stitch (AS), As-natural-as-possible (ANAP), Xiaomin's result.

But in our proposed method, an optimal seam is chosen by dynamic programming and a mask is created for moving objects. With this information, the overlap regions are updated, and image pairs are blended along the seam using mask of possible moving objects.

Moreover, we checked experimentally the proposed method performance for images that have parallax artifacts. As described in Fig. 9 two input images are stitched by applying different methods. Due to existence of parallax in input images, most of the methods fail to generate an appropriate result. In the result of PM dislocation of train rails are clearly visible along stitching line. And there are disposition errors of train rails and increased number of blurred rails on the result of AS and Xiaomin's method. For handling parallax artifacts and reducing perspective distortion ANAP uses a local hommography and global similarity transformation. On the result of ANAP parallax errors are eliminated but this process is timeconsuming process and needs additional step to avoid transition seams. However, there is smaller dislocation error on the result of the proposed method compared to AS, PM, Xiaomin's methods. Because, the proposed method updates overlap regions using an optimal seam line 
and applies blending technique. In the case of having large parallax the proposed method may not achieve satisfactory result. Because the method is good at stitching images that are taken in real-time cases by rotating a camera or a smartphone.

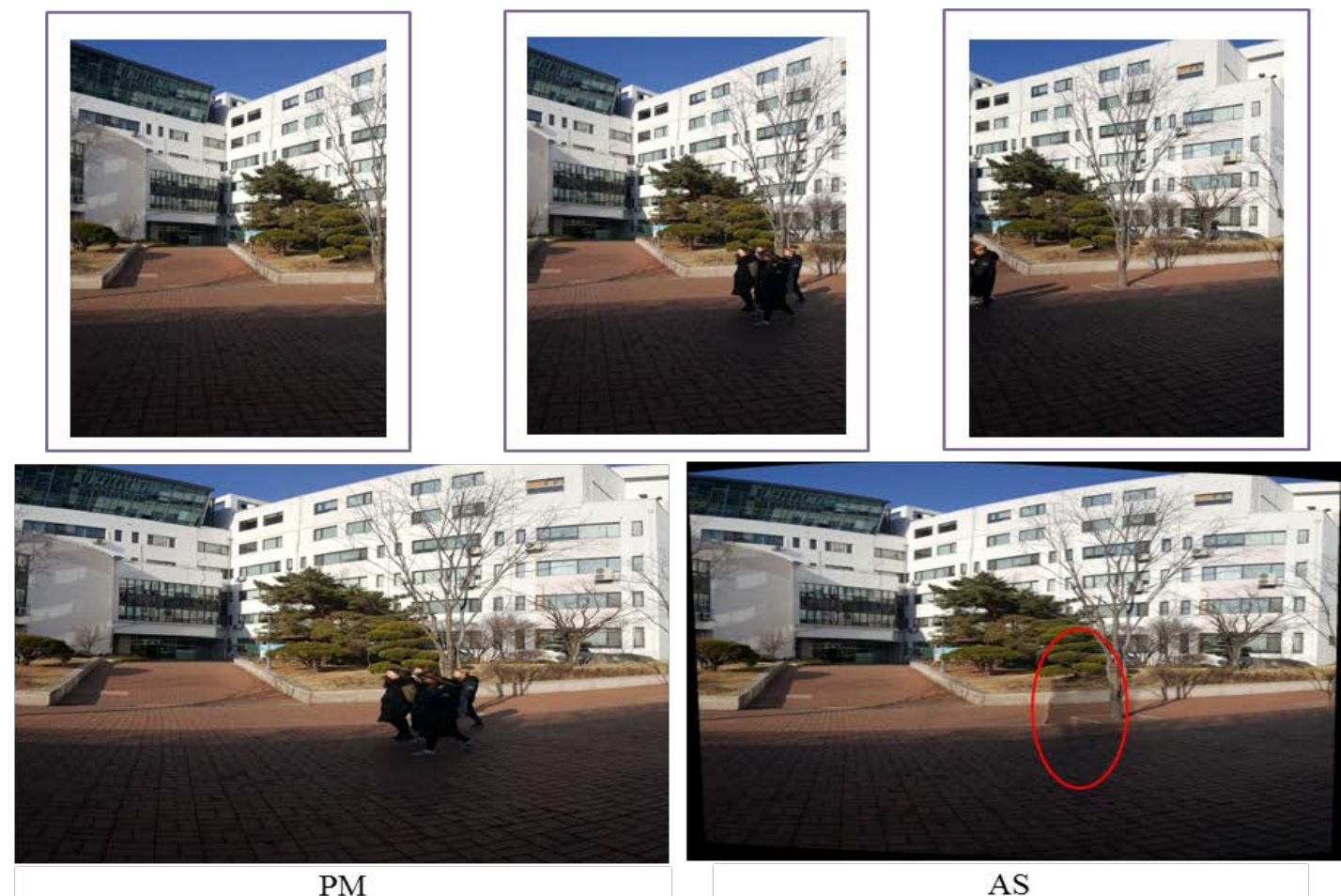

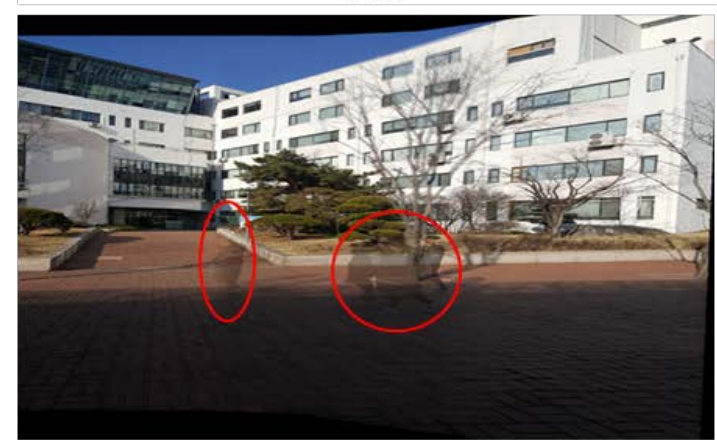

Xiaomin's result

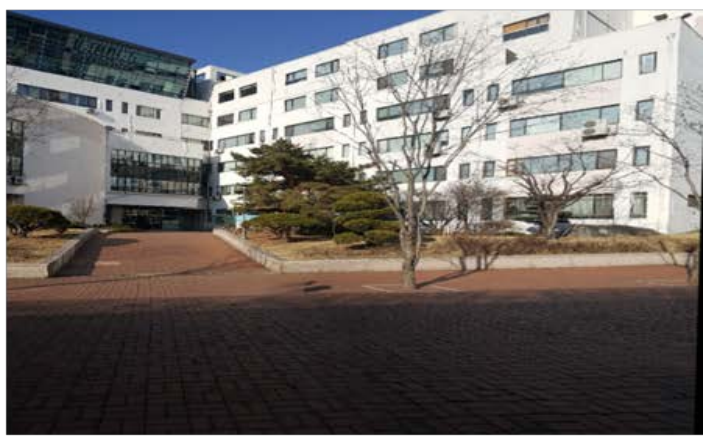

Our result

Fig. 8. Comparisons between our result and other state-of-the-art methods' results, Panorama Maker (PM), Auto Stitch (AS), Xiaomin’s result.

In addition, we compared the performance of the proposed method with the state-of-the-art methods in terms of speed. As illustrated in Table 1, ANAP method takes more time compared to others do. Because, it uses a local hommgraphy with global similarity transformation. In case there are large overlap regions,(for Fig. 7) it spends so much time to find optimal global similarity and local transformation. Furthermore, it has a visible transition seams after stitching images. Among these methods Xiaomin's method stitches images fastly. But the method does not have a technique of seam estimation and the blending technique to avoid the ghosting in the presence of moving objects, small parallax. Due to the presence of 
moving objects, AS also has ghosting artifacts on the result. But the proposed method achieves high speed performance and handles moving object artifacts.

Furthermore, using image-cropping techniques, we can obtain rectangular boundary panoramic images.

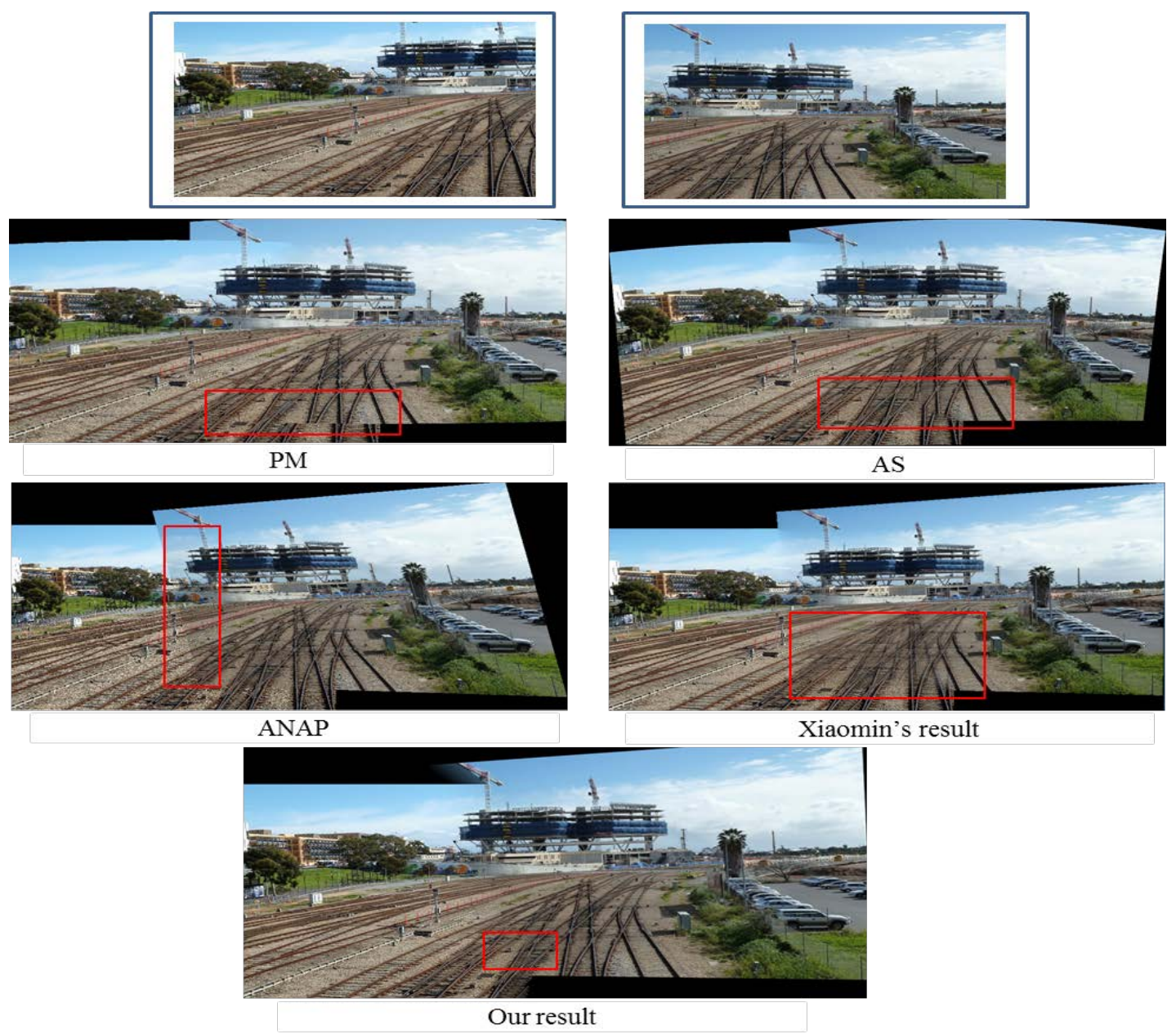

Fig. 9. Comparisons between our result and other state-of-the-art method results for images having parallax, Panorama Maker (PM), As-natural-as-possible (ANAP), Auto Stitch (AS) and Xiaomin's result.

One weakness of the method we used a combination of linear blending with mask information on purpose of seamless blending efficiently. However, when different high luminance affects on input images the method may produce inapplicable result. Because linear blending suits the requirements of realtime applications on low-powered devices and smarphones but, it may not remove transition seams completely. 
Table 1. Performance analysis of the method with existing methods

\begin{tabular}{|c|c|c|c|}
\hline \multirow{2}{*}{$\begin{array}{c}\text { Stitching } \\
\text { methods }\end{array}$} & \multicolumn{3}{|c|}{ Processing time } \\
\cline { 2 - 4 } & Fig. 7 & Fig. 8 & Fig. 9 \\
\hline \hline AutoStitch & 2.62 & 8.89 & 2.55 \\
\hline $\begin{array}{c}\text { Panorama } \\
\text { Maker }\end{array}$ & 2.36 & 6.51 & 2.26 \\
\hline ANAP & 14.56 & - & 2.74 \\
\hline Xiaomin & 1.16 & 4.04 & 1.01 \\
\hline Our method & 1.33 & 4.84 & 1.16 \\
\hline
\end{tabular}

\section{Conclusion}

This paper proposed a new method for creating panoramic images in the presence of moving objects. We used one of the fastest feature extraction method, dynamic programming for finding optimal seams, linear blending method with the mask information and image-cropping techniques. First, unique and robust feature points are extracted by applying ORB method. Then, corresponding feature points are matched and all parameters of the transformation matrixes are calculated using RANSAC algorithm. Following that, a sequence of techniques for image correction has been applied to find an optimal seam and create a mask for possible moving objects. Finally, a combination of linear blending with the mask information is utilized to produce a seamless panoramic image without ghosting artifacts and image-cropping technique is employed. From the experiments, our method addresses the ghosting artifacts caused by dynamic objects and it has good speed to produce a pleasing panoramic image with rectangular boundaries.

Future work includes improving the quality of panoramic images for images that have high illumination changes.

\section{References}

[1] Abdukholikov Murodjon, Taeg-keun Whangbo, "A method for manipulating moving objects in panoramic image stitching," in Proc. of 2017 International conference on Emerging Trends \& Innovation in ICT(ICEI), India, 3-5 Feb., 2017. Article (CrossRef Link)

[2] Yingen Xiong, Nokia Research Center, "Eliminating ghosting artifacts for panoramic images," 2013 IEEE International Symposium on Multimedia, pp.432-437, 2009. Article (CrossRef Link)

[3] Ebtsam Adel, Mohammed Elmogy, Hazem Elbakry, "Image stitching system based on ORB feature based technique and compensation blending," (IJACSA) International Journal of Advanced Computer Science and Applications, vol. 6, no. 9, 2015. Article (CrossRef Link)

[4] D. G. Lowe, "Distinctive image features from scales-invariant keypoints," International Journal of Computer Vision, vol. 60, no. 2, pp. 91-110, 2004. Article (CrossRef Link)

[5] Bay, Herbert, Andreas Ess, Tinne Tuytelaars, and Luc Van Gool. Speed-Up Robust Features (SURF) Comput. Vis. Image Underst. -New York, NY, USA: Elsevier Science Inc., vol. 110, no. 3, pp. 346-359. -ISSN: 1077-3142, jun. 2008. Article (CrossRef Link)

[6] Rublee, Ethan, Vincent Rabaud, Kurt Konolige, and Gary Bradski. ORB: An Efficient Alternative to SIFT or SURF. Proceedings of the 2011 International Conference on Computer Vision. Washington, DC, USA: IEEE Computer Society, pp. 2564-2571. - ISBN: 978-1-4577-1101-5, 2011. Article (CrossRef Link)

[7] Wuxia Yan, Chuancai Liu, Wei Luo, "Fast and low complexity image stitching method on mobile phones," in Proc. of Control, Automation and Information Sciences(ICCAIS), 2015 International Conference, 2015. Article (CrossRef Link) 
[8] Yanli Wan and Zhenjiang Miao, “Automatic panorama image mosaic and ghost eliminating,” in Proc. of Multimedia and Expo, 2008 IEEE International Conference on., IEEE, pp.945-948, 2008. Article (CrossRef Link)

[9] Jun-Wei Hsieh, "Fast stitching algorithm for moving object detection and mosaic construction," Image and Vision Computing, vol. 22, pp 291-306, 2004, Article (CrossRef Link)

[10] Jisung Yoo, Sung Soo Hwang, Seong Dae Kim, Myung Seok Ki, Jihun Cha,"Regional Linear Warping for Image Stitching with Dominant Edge Extraction,” KSII Transactions on Internet and Information Systems, vol. 7, no. 10, pp. 2464-2478, 2013, Article (CrossRef Link)

[11] Chung-Ching Lin, Sharath Pankanti, Karthikeyan Natesan Ramamurthy, and Aleksandr Y. Aravkin, “Adaptive As-Natural-As-Possible Image Stitching,” in Proc. of 2015 IEEE Conference on Computer Vision and Pattern Recognition (CVPR), 2015. Article (CrossRef Link)

[12] Qingpeng Chai, Shiguang Liu, “Shape-optimizing hybrid warping for image stitching,” in Proc. of 2016 IEEE International Conference on Multimedia and Expo (ICME), pp. 1-6, 2016. Article (CrossRef Link)

[13] Xiaomin Ma, Ding Liu, Jian Zhang, Jing Xin, “A fast affine-invariant features for image stitching under large viewpoint changes,” Neurocomputing, vol. 151, no. 3, pp. 1430-1438, 2015. Article (CrossRef Link)

[14] A. Alahi, R. Ortiz, P. Vandergheynst, "FREAK: Fast Retina KeyPoint," in Proc. of the IEEE Conference on Computer Vision and Pattern Recognition(CVPR), pp. 510-517, 2012. Article (CrossRef Link)

[15] Yu-jing Zhao, Zhe-qi Lu and Yang-ke Liu, "Video image stitching based on moving object detection and motion prediction compensation," Image and Signal Processing(CISP), 20103 rd International Congress, vol. 6, pp. 2885-2889, 2010. Article (CrossRef Link)

[16] J. Davis, "Mosaics of scenes with moving objects," in Proc. of CVPR 98: Proceedings of the IEEE Computer Society Conference on Computer Vision and Pattern Recognition. Washington, DC, USA: IEEE Computer Society, p.354, 1998. Article (CrossRef Link)

[17] Alec Mills, Gregory Dudek, "Image stitching with dynamic elements," Image and Vision Computing, vol. 27, issue 10, pp. 1593-1602, 2009. Article (CrossRef Link)

[18] Edsger W. Dijkstra, “A note on two problems in connexion with graphs,” Numerische Matematik, pp. 269-271, 1959. Article (CrossRef Link)

[19] M. Kazhdan and H.Hoppe, "Streaming multigrid for gradient-domain operations on large images," ACM Trans. Graph, vol. 27, no. 3, pp. 1-10, 2008. Article (CrossRef Link)

[20] K. Ram Prabhakar, R. Venkatesh Babu "Ghosting-free multi-exposure image fusion in gradient domain” in Proc. of Acoustics, Speech and Signal Processing (ICASSP), 2016 IEEE International Conference, Article (CrossRef Link)

[21] Yingen Xiong, Kari Pulli "Gradient Domain Image blending and Implementation on Mobile Devices,” in Proc. of MobiCase '09: The First Annual International Conference on Mobile Computing, Applications, and Services', USA, 2009. Article (CrossRef Link)

[22] P. Perez, M. Gangnet, and A. Blake, “Poisson image editing,” ACM Trans Graph.,vol. 22, no. 3, pp. 313-318, 2003, Article (CrossRef Link)

[23] J. Jia, J. Sun, C.-K. Tang, and H.-Y. Shum, “Drag-and-drop pasting,” in SIGGRAPH '06: ACM SIGGRAPH 2006 Papers, New York, NY, USA: ACM, pp.631-637, 2006. Article (CrossRef Link)

[24] P.J. Burt, E.H. Adelson, "Multiresolution spline with application to image mosaics," ACM Transactions on Graphics, vol. 2, no. 4, pp. 217-236, 1983, Article (CrossRef Link)

[25] S. Ha, H. Koo, S. Lee, N. Cho and S. Kim, "Panorama mosaic optimization for mobile camera systems,” IEEE Transactions on Consumer Electronics, vol. 53, no. 4, pp. 1217-1225, Nov. 2007, Article (CrossRef Link)

[26] Xiaoqing Yin, Weili Li, Bin Wang, Yu Liu and Maojun Zhang, "A Novel Video Stitching Method for Multi-Camera Surveillance Systems,” KSII Transactions on Internet and Information Systems, vol. 8, no. 10, pp.3538-3556, 2014. Article (CrossRef Link) 
[27] S. Ha, H. Koo, S. Lee, N. Cho and S. Kim, "Panorama mosaic optimization for mobile camera systems,” IEEE Transactions on Consumer Electronics, vol. 53, no. 4, pp. 1217-1225, Nov. 2007, Article (CrossRef Link)

[28] A. A. Efros and W. T. Freeman, "Image quilting for texture synthesis and transfer," ACM SIGGRAPH, pp. 341-346, 2001, Article (CrossRef Link)

[29] Y. Boykov, O. Veksler and R. Zabih. "Fast approximate energy minimization via graph cuts," IEEE Transactions on Pattern Analysis and Machine Intelligence, vol. 23, no. 11, pp. 1222-1239, 2001, Article (CrossRef Link)

[30] S. N. Sinha, J. Michael Frahm, M. Pollefeys, and Y. Genc. "GPU-based video feature tracking and matching," Technical report, In workshop on Edge Computing Using New Commodity Architectures, 2006, Article (CrossRef Link)

[31] E. Rosten and and T. Drummond. "Machine Learning for high speed corner detection.," In European Conference on Computer Vision, vol. 1, 2006, Article (CrossRef Link)

[32] M. Calonder, V. Lepetit, C. Streacha, and P. Fua. "BRIEF: Binary robust independent elementary features,” in Proc. of European Conference on Computer Vision, 2010. Article (CrossRef Link)

[33] P. L. Rosin, "Measuring corner properties," Computer Vision and Image Understanding, vol. 73, no. 2, pp. 291-307, 1999. Article (CrossRef Link)

[34] M. Brown and D. G. Lowe, "Automatic panoramic image stitching using invariant features," International Journal of Computer Vision, vol. 74, no. 1, pp. 59-73, 2007. Article (CrossRef Link)

[35] Panorama Maker, https://panorama-maker.en.softonic.com

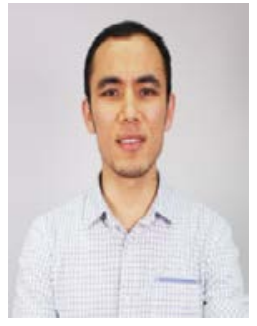

Abdukholikov Murodjon received the B.S. degree from Tashkent University of Information Technologies, Tashkent, Uzbekistan in 2015 and he is currently studying for a M.S. degree in IT Convergence Engineering at Gachon University, Korea. His research interests include Computer Vision, Virtual Reality, Big Data, Sentiment Analysis and Artificial Intelligence.

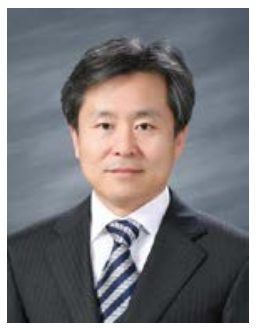

Taeg Keun Whangbo received the M.S. degree from City University of New York in 1988 and the Ph.D. degree both in Computer Science from Stevens Institute of Technology in 1995. Currently, he is a professor in the Department of Computer Science, Gachon University, Korea. Before he joined the Gachon University, he was the software developer in Q-Systems which is located in New Jersey from 1988 to 1993. He was also the researcher in Samsung Electronics from 2005 to 2007. From 2006 to 2008, he was the president of the Association of Korea Cultural Technology. His research areas include Computer Graphics, HCI and VR/AR. 